\title{
SENSITIVITY AND SPECIFICITY OF THREE-POINT COMPRESSION ULTRASONOGRAPHY TEST PERFORMED BY EMERGENCY PHYSICIANS FOR DIAGNOSIS OF LOWER
}

LIMBS DEEP VENOUS THROMBOSIS

Assem Abd-Elrazek Abd-Rabo ${ }^{1}$, Tamer Abdullah Helmi ${ }^{2}$, Hassan Abd-Elsalam Fathi ${ }^{3}$, Asmaa Mohamed Alkafafy ${ }^{4}$, Eman Helmi Ahmed El-Gazzar ${ }^{4}$

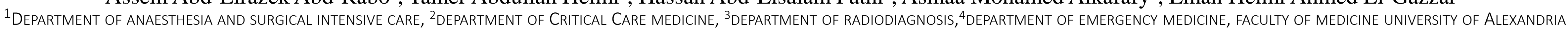

\section{INTRODUCTION:}

$>$ Deep vein thrombosis (DVT) is defined as development of thrombosis within

the deep veins of the pelvis or lower limbs.

Thrombus formation is a dynamic, multifactorial process that depends on a fine three main causes: vessel wall injury, hypercoagulability, and venous stasis which is known as Virchow's triad.

Early detection of deep venous thrombosis is very important not only to initiate anticoagulant therapy, but also very important to prevent further complications and to reduce notidity and mortality.

作

Development of DVT is affected by many factors either genetic or acquired risk factors like age, Trauma, Cancer, Surgery, Pregnancy, Obesity, Immobility, states, anaesthesia, myocardial infarction, history of DVT, varicose veins, infections, inflammatory bowel disease, dehydration, and renal impairment are common risk factor

$>$ Compression ultrasonography is the first-line imaging tool for diagnosis of DVT because it is safe,

It is accurate to determine the size chronicity

and degree of occlusion of a thrombus which is

very helpful for making the decision of starting

medical management or interventional techniques.

\section{AIM OF THE WORK}

> The aim of this study was to evaluate the sensitivity and specificity of the threepoint compression ultrasonography test performed by emergency medicine residents at the emergency department of Alexandria Main University Hospital for early detection of lower limbs deep vein thrombosis in an expected shorter duration in comparison to the formal doppler ultrasonography by radiology residents.

\section{SUBJECT:}

> This study included 100 patients collected from (AMUH) Emergency Department, according to a sample size calculation done at the "Community Medicine Department, Faculty of Medicine, Alexandria University".

\section{Inclusion criteria:}

$>$ Age $>18$ years

Any patient complaining of acute unilateral or bilateral limb swelling. - Suspected DVT according to Well's score for diagnosis of DVT.

Exclusion criteria

Long bone fracture at the affected limb.

Chronic lower limb swelling.

Patients with chronic peripheral vascular diseases.

> Patients who have an inserted femoral catheter.

$>$ Morbid obesity (body mass index 40 or more)

$>$ Sever lower limb pain, agitation.

\section{METHODS:}

Study Design: prospective cross-sectional study.

1. Data Collection: Complete history taking; age, sex, medical, drug.. etc.

2. General physical examination: according to the $\mathrm{ABCDE}$ approach,

Airway; maintain a patent, clear and secure airway.

Breathing; maintain breathing, oxygenation and respiratory rate.

Circulation; blood pressure, mean arterial pressure, heart rate.

Disability; Glasgow coma scale, random blood sugar and pupils assessment.

Exposure and general examination (head to toe examination).

Local examination of the suspected limb and Wells score (signs of DVT)

Unilateral or bilateral lower limb edema.

Hotness at the affected side.

Tender calf muscles.

Motor sensory functions.

Presence or absence of skin changes; ulcers or discoloration

Laboratory investigations:

Complete blood picture, prothrombin time, International normalized ratio, Serum creatinine, D-Dimer.

5. Emergency department three-point compression test:

Ultrasound examination of 3 sites: common femoral vein, sapheno-femoral

junction, popliteal vein.

Methods to distinguish the artery from the vein: The artery is minimally compressible while the vein is fully compressible, it is larger than the artery, and will demonstrate respiratory collapse.

6. Formal radiological ultrasound examination.
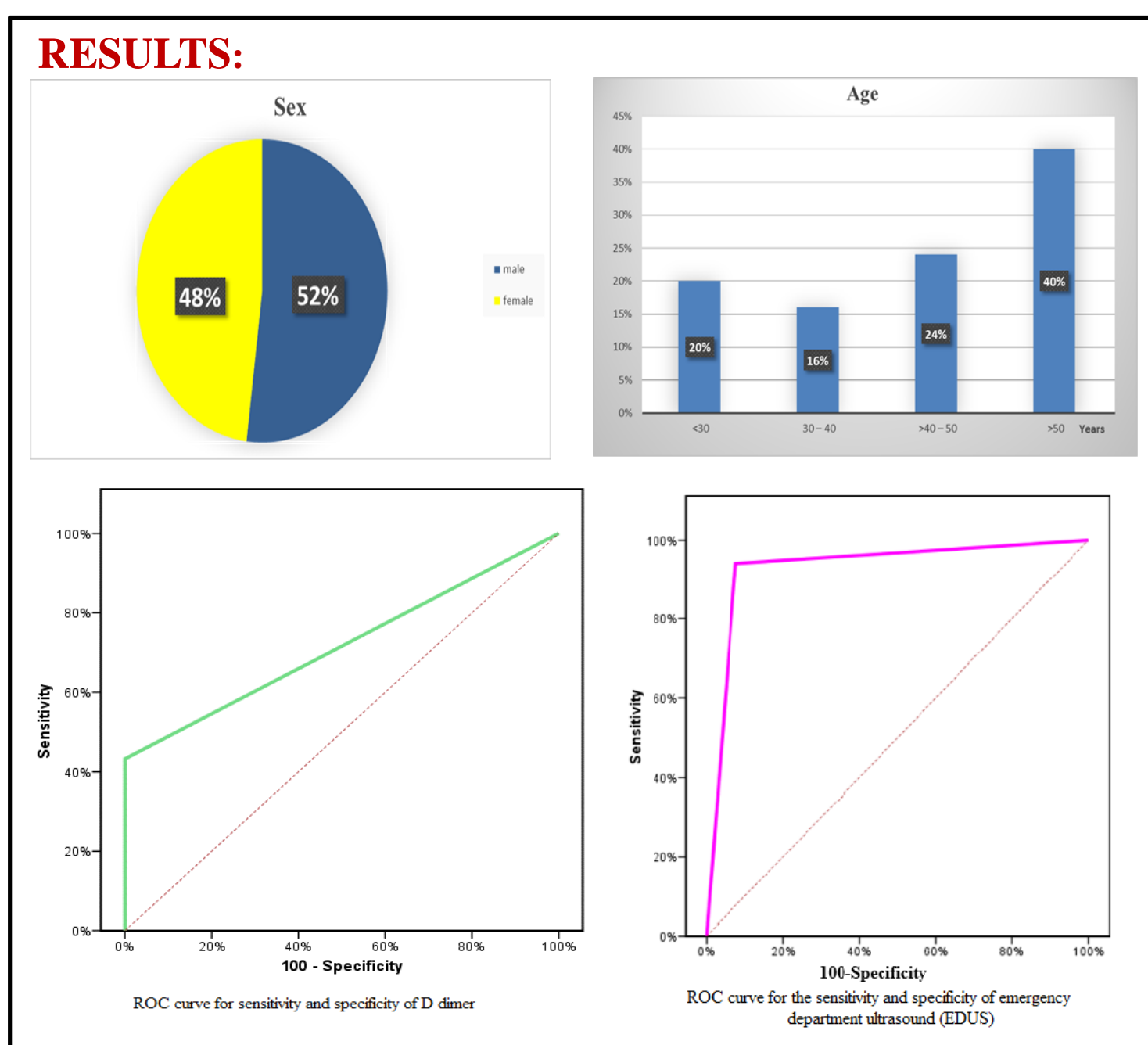

CONCLUSION:

$>$ The emergency department ultrasound can be conducted after shorter The emor ultrasound is highly sensitive $(94.12 \%)$, highly specific $(92.42 \%)$ and very accurate $(93.0 \%)$ diagnostic tool. No statistically
significant difference between the results of ultrasound done by significant difference between the results of ultrasound done by
emergency physician and that done by radiology resident.

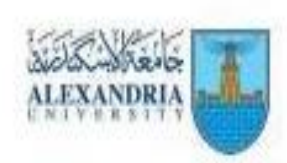

2019@Alexandria Faculty of Medicine MECULTY OF 